\title{
Serum Lp-PLA2 Level Predicting Coronary Artery Lesions in Children with Kawasaki Disease
}

\author{
Xiong Zhang, ${ }^{1}$ Ya-Wang Shao, ${ }^{2}$ Ya-Lan Zhang, ${ }^{1} \mathrm{Yi} \mathrm{Liu}^{3}$ \\ ${ }^{1}$ Department of Laboratory, First People's Hospital of Xianyang City, Xianyang, Shaanxi Province, China; \\ ${ }^{2}$ Department of Pediatrics, First People's Hospital of Xianyang City, Xianyang, Shaanxi Province, China; \\ ${ }^{3}$ Department of Laboratory, First Affiliated Hospital of Xi'an Jiaotong University, Shaanxi Province, China
}

\section{ABSTRACT}

Background: Kawasaki disease (KD) is an inflammatory disease associated with coronary vasculitis in children. In this study, we explored the correlation between Lipoprotein associated phospholipase A2 (Lp-PLA2) and coronary artery lesions (CAL) in children with KD.

Methods: Ninety-three children with KD were divided into a normal coronary artery (NCA, 54 cases) group and coronary artery lesions (CAL, 39 cases) group, according to the results of echocardiography. Another 42 healthy children were selected as the control group. The serumal levels of Lp-PLA2, Interferon- $\gamma($ IFN- $\gamma$ ) and Interleukin-6 (IL-6) were determined by using an enzyme-linked immunosorbent assay. In addition, erythrocyte sedimentation rate (ESR) and serum C-reactive protein (CRP) level were analyzed. The left main coronary artery (LMCA), diameters of left anterior descending coronary artery (LADC), right proximal coronary artery (PRCA), and carotid intima-media thickness (IMT) were obtained by color Doppler ultrasound. The correlation between the above indexes and $\mathrm{KD}$ was analyzed.

Results: The levels of white blood cell counts (WBC), ESR, CRP, IFN- $\gamma$, IL-6 and Lp-PLA2 as well as IMT were significantly increased in KD children $(P<0.05)$, and the levels of CRP, IFN- $\gamma$, IL-6 and Lp-PLA2 as well as IMT in the CAL group increased more significantly $(P<0.05)$. An increasing trend also has been described in the diameters of LMCA, LADC and PRCA for KD children with CAL compared with with NCA. The results of logistic regression analysis showed that the elevated levels of CRP, IFN- $\gamma$, IL-6 and Lp-PLA2 were independent risk factors for $\mathrm{KD}$ with CAL. Correlation analysis showed that Lp-PLA2 level was positively correlated with the levels of IFN- $\gamma$, IL-6 and CRP in CAL group and NCA group (respectively, all $P<0.01$ ). In addition, a similar correlation was also described between Lp-PLA2 level and the diameters of LMCA, LADC and PRCA in CAL group (respectively, all $P<0.01$ ).

Received March 26, 2021; accepted May 10, 2021.

Correspondence: Ya-Lan Zhang, Department of Laboratory, First People's Hospital of Xianyang City, No. 10 Biyuan West Road, Xianyang City, Shaanxi Province, Postal Code 712000, China (e-mail: 1781292176@qq.com)
Conclusion: Lp-PLA2 may participate in the pathological mechanism of KD. Detection of the serum Lp-PLA2 level can be used in the diagnosis of KD disease and the assessment of coronary artery lesions in KD children.

\section{INTRODUCTION}

Kawasaki disease (KD) is a kind of inflammatory disease associated with coronary vasculitis in children that mainly involves medium-sized arteries. The main symptoms are fever, conjunctivitis, skin and mucosa lesions, cervical lymph node enlargement [Hedrich 2018]. It is the most common cause of child-acquired heart disease in developed countries. At present, the etiology of KD is not clear [Menikou 2019]. A study has shown that KD may be complicated with aneurysms, coronary thrombosis, and myocardial infarction [McCrindle 2017]. Early diagnosis and treatment can improve the prognosis. Lipoprotein associated phospholipase A2 (Lp-PLA2), as a marker of vascular inflammation, is closely related to the occurrence and development of atherosclerotic cardiovascular disease [Choi 2018]. By analyzing the changes of serum Lp-PLA2 level in children with $\mathrm{KD}$, this study aims to explore the correlation between Lp-PLA2 and coronary artery lesions (CAL) in children with $\mathrm{KD}$ and to provide theoretical basis for clinical diagnosis and treatment of KD.

\section{MATERIALS AND METHODS}

General data: Ninety-three children with KD admitted to the First Affiliated Hospital of Xi' an Jiaotong University from January 2015 to January 2019 were divided into two groups - the normal coronary artery (NCA) group $(N=54)$ and coronary artery lesions (CAL) group $(N=39)$ on the basis of the results of echocardiography. In the CAL group, the diameter of coronary artery was $\geq 2.5 \mathrm{~mm}$ in children younger than 3 years old, $\geq 3.0 \mathrm{~mm}$ in children age $3-9$ years old, and $\geq 3.5$ $\mathrm{mm}$ in children over 9 years. In the NCA group, the diameter of coronary artery was less than $2.5 \mathrm{~mm}$ in children under 3 years old, less than $3.0 \mathrm{~mm}$ in children between 3 and 9 years old, and less than $3.5 \mathrm{~mm}$ in children over 9 years. The CAL group included 30 patients with small coronary artery aneurysms (coronary artery diameter $\leq 4 \mathrm{~mm}$ ), five patients with middle coronary artery aneurysms (coronary artery diameter 
between 4 and $8 \mathrm{~mm}$ ), and four patients with giant coronary artery aneurysms (coronary artery diameter $>8 \mathrm{~mm}$ ).

Exclusion criteria included infectious mononucleosis, sepsis, scarlet fever, Steven Johnson syndrome, with typical cardiovascular complications. Another 30 healthy children in the same period were selected as the control group. There was no significant difference in gender, age, and body mass index among the three groups $(P>0.05)$. (Table 1)

Diagnosis of disease: The diagnostic criteria of Kawasaki disease refer to the 2017 AHA KD diagnostic guidelines [McCrindle 2017]. The diagnostic criteria of coronary artery dilatation refer to the clinical treatment recommendations of Kawasaki disease complicated with coronary artery disease in 2012 [Biscetti 2019].

Methods: The clinical data of participants were collected, including gender, age, and body mass index. The serumal levels of Lp-PLA2, IFN- $\gamma$ and IL-6 were determined by using an enzyme-linked immunosorbent assay method, according to manufacturers' specifications (Lp-PLA2: kangerke Biotechnology Co., Ltd., Tianjin, China; IFN- $\gamma$ and IL-6: R\&D Systems, Minneapolis, MN, USA). Routine blood tests were performed by Sysmex XN-2000 instrument (Sysmex, Kobe, Japan). ESR was performed with a commercially available kit using the Wintrobe method (Winpette, Guest Scientific AG, Switzerland). CRP level was measured by an immunoturbidimetric assay using a Modular P800 automatic analyzer (Roche Diagnostics GmbH, Mannheim, Germany).

Specimen collection: Eight $\mathrm{ml}$ of fasting venous blood were collected from all participants and $5 \mathrm{ml}$ were centrifuged at $3000 \mathrm{R} / \mathrm{min}$ for $10 \mathrm{~min}$. The serum was separated and stored at $-20^{\circ} \mathrm{C}$ for analysis of CRP, IFN- $\gamma$, IL-6, and Lp-PLA2. Another $3 \mathrm{ml}$ were used for white blood cell counts (WBC) and ESR analysis.

Color Doppler ultrasound analysis: All participants underwent routine echocardiography (Vivid7 Dimension, General Electric Company, Boston, MA, USA). When the children were asleep or in a quiet state, the standard cross sections of the heart in the infraxiphoid fossa, apical fossa, parasternal fossa, and suprasternal fossa routinely were explored, and the diameters of left main coronary artery (LMCA), left anterior descending coronary artery (LADC) and right proximal coronary artery (PRCA) were measured. If coronary artery aneurysms were found, the diameter and length of the aneurysms were measured (at least three times, the average value was taken), and the thrombosis was noted.

Carotid ultrasonography: One year after the onset of $\mathrm{KD}$, the children underwent carotid ultrasound examination. Color Doppler ultrasound was used to observe the vascular condition of the left and right common carotid artery trunk $(2 \mathrm{~cm}$ before bifurcation), carotid bifurcation and internal carotid artery (1 $\mathrm{cm}$ before bifurcation). The posterior wall of the artery showed a "double line" image. The inner line was the boundary between the intima and lumen, and the outer line was the boundary between the medial and adventitia. The average value of the three points on the left and right sides was taken as the left and right IMT, respectively, and the larger value on both sides was taken as the IMT. The IMT data of KD children were compared with those of age and gender matched normal controls.

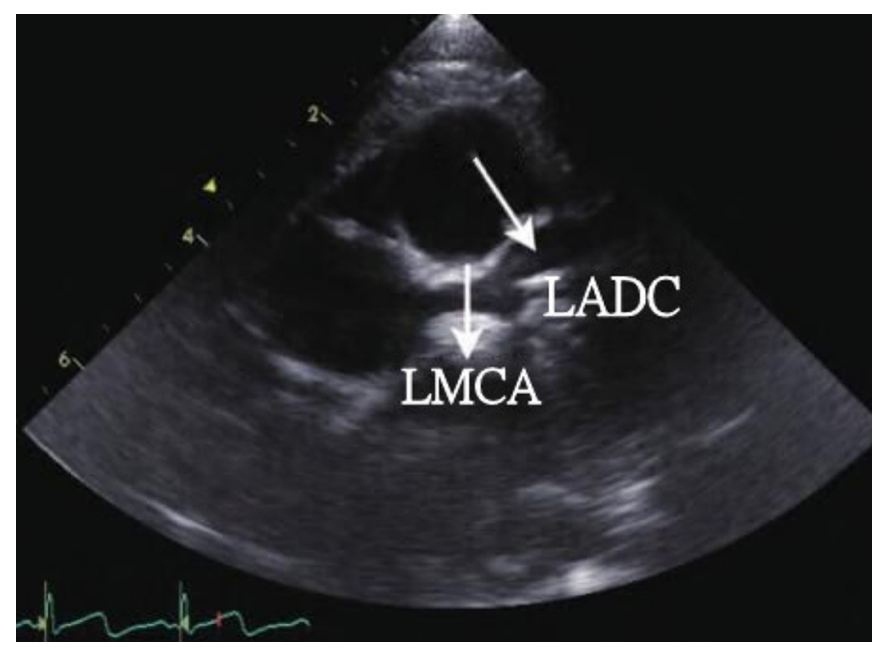

Figure 1. Left coronary artery dilatation. LMCA, left main coronary artery; LADC, left anterior descending coronary artery

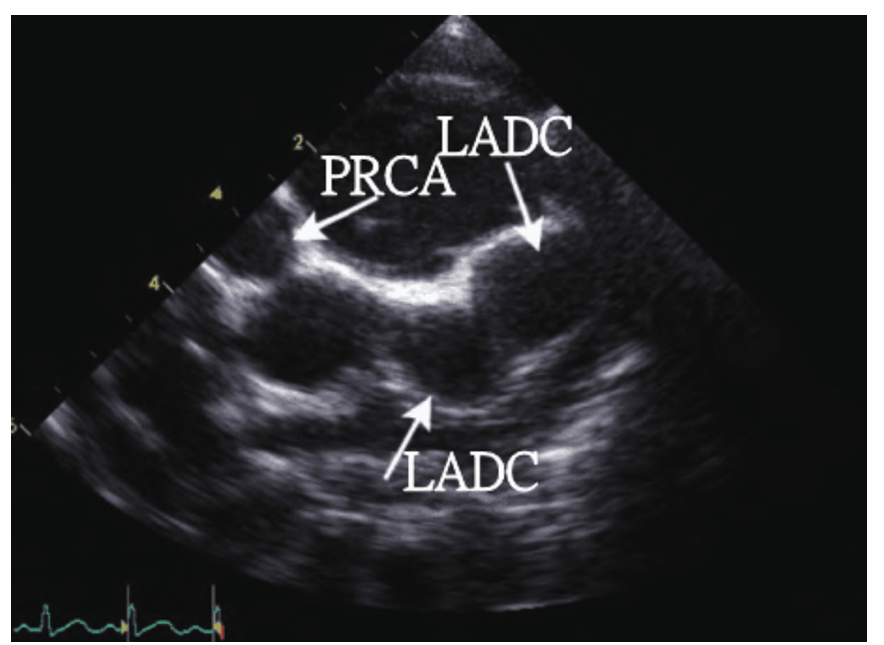

Figure 2. Giant aneurysm-like dilation of left and right coronary arteries in children with KD. LMCA, left main coronary artery; LADC, left anterior descending coronary artery; PRCA, right proximal coronary artery

Statistical analysis: Graphpad Prism 5 (GraphPad Software, Inc., La Jolla, Calif., USA) was used to analyze the data. The normality test was analyzed by KolmogorovSmirnov. The measurement data of normal distribution was expressed as mean \pm Standard deviation $\mathrm{v} \pm \mathrm{s}$ ). Measurement data were compared by independent sample t-test, one-way ANOVA with post-hoc Tukey's test. Count data were compared by chi square test. Correlation analysis was performed by Pearson method. Logistic regression was used to establish the relationship model between dependent variables and risk factors, and the results were expressed as an odds ratio (OR) with a 95\% confidence interval (CI). Receiver operating characteristic curve (ROC curve) was established to evaluate the predictive value of Lp-PLA2 in the diagnosis of $\mathrm{KD}$ or with CAL. The difference was statistically significant $(P<0.05)$. 
Table 1. Comparison of general data among the groups

\begin{tabular}{lcccc}
\hline Items & Control group $(N=30)$ & NCA group $(N=54)$ & CAL group $(N=39)$ & $P$-value \\
\hline Male (cases) & 18 & $33^{*}$ & $24^{*} \#$ & $>0.05$ \\
Age $\left(\bar{x}_{ \pm s}\right.$, months $)$ & $36.29 \pm 13.53$ & $35.32 \pm 14.12$ & $35.66 \pm 13.97^{*} \#$ & $>0.05$ \\
Body mass index $\left(\bar{x}_{ \pm s,} \mathrm{~kg} / \mathrm{m} 2\right)$ & $14.63 \pm 3.19$ & $14.36 \pm 3.02 *$ & $14.15 \pm 3.37^{*} \#$ & $>0.05$ \\
\hline
\end{tabular}

$* P$ value $>0.05$, Compared with the control group; $\# P$ value $>0.05$, Compared with NCA group

Table 2. Comparison of coronary artery diameters among three groups $\left({ }^{{ }} \pm s, \mathrm{~mm}\right)$

\begin{tabular}{lcccc}
\hline Items & Control group $(N=30)$ & NCA group $(N=54)$ & CAL group $(N=39)$ & $P$-value \\
\hline LMCA & $2.48 \pm 0.23$ & $2.51 \pm 0.23$ & $3.12 \pm 0.25^{*} \#$ & $<0.01$ \\
LADC & $2.30 \pm 0.21$ & $2.34 \pm 0.20$ & $2.95 \pm 0.23^{*} \#$ & $<0.01$ \\
PRCA & $2.29 \pm 0.20$ & $2.33 \pm 0.21$ & $2.91 \pm 0.22^{*} \#$ & $<0.01$ \\
IMT & $0.420 \pm 0.017$ & $0.447 \pm 0.020^{*}$ & $0.471 \pm 0.024^{*} \#$ & $<0.05$ \\
\hline
\end{tabular}

$* P$ value $<0.05$, Compared with the control group; \#P value < 0.05, Compared with NCA group. CAL, coronary artery lesions; NCA, normal coronary artery; LMCA, left main coronary artery; LADC, left anterior descending coronary artery; PRCA, right proximal coronary artery; IMT, carotid intima-media thickness

Table 3. Comparison of coronary artery diameters in different age groups between the CAL group and control group $\left({ }^{\bar{x}} \pm s, \mathrm{~mm}\right)$

\begin{tabular}{|c|c|c|c|c|c|}
\hline \multirow[t]{2}{*}{ <3岁 } & Control group & 18 & $2.26 \pm 0.17$ & $2.09 \pm 0.15$ & $2.08 \pm 0.16$ \\
\hline & $P$-value & - & $<0.01$ & $<0.01$ & $<0.01$ \\
\hline \multirow[t]{2}{*}{ 3 9岁 } & Groups & 10 & $2.73 \pm 0.22$ & $2.55 \pm 0.20$ & $2.53 \pm 0.20$ \\
\hline & $P$-value & - & $<0.01$ & $<0.01$ & $<0.01$ \\
\hline \multirow[t]{3}{*}{$>9$} & Control group & 2 & $3.19 \pm 0.25$ & $3.01 \pm 0.23$ & $2.99 \pm 0.21$ \\
\hline & CAL group & 3 & $3.97 \pm 0.26$ & $3.83 \pm 0.25$ & $3.81 \pm 0.25$ \\
\hline & $P$-value & - & $<0.01$ & $<0.01$ & $<0.01$ \\
\hline
\end{tabular}

CAL, coronary artery lesions; NCA, normal coronary artery; LMCA, left main coronary artery; LADC, left anterior descending coronary artery; PRCA, right proximal coronary artery; IMT, carotid intima-media thickness

\section{RESULTS}

Echocardiographic diagnosis analysis for KD: Among the 93 children with Kawasaki disease, 54 cases $(58.07 \%)$ in the NCA group and 39 cases $(41.93 \%)$ in the CAL group were detected by echocardiography. In the CAL group, there were 14 cases of left coronary artery dilatation, nine cases of right coronary artery dilatation, 12 cases of bilateral coronary artery dilatation, and four cases of giant coronary artery aneurysm (three cases were located in the initial segment of right coronary artery and 1 case was located in the initial segment of left coronary artery). The left coronary artery dilation was shown in Figure 1. (Figure 1) The diameter of giant coronary artery aneurysm was shown in Figure 2. (Figure 2)

The diameters of LMCA, LADC and PRCA in the CAL group were significantly higher than those in the NCA group and control group $(P<0.05)$, and a similar trend was described from the different age groups between the CAL group and control group $(P<0.05)$, but there were no significant differences between the NCA group and control group $(P>0.05)$. Moreover, IMT of the control group, NCA group and CAL group also showed a gradually increasing trend $(P<0.05)$. (Table 2) (Table 3) (Figure 3) 
Laboratory analysis for KD: The levels of WBC, ESR, CRP, IFN- $\gamma$, IL-6, and Lp-PLA2 were significantly increased in KD children $(P<0.05)$, and the levels of CRP, IFN- $\gamma$, IL-6 and Lp-PLA2 in the CAL group increased more significantly $(P<0.05)$ (Figure 3). (Table 4)

Logistic regression: Logistic regression analysis showed that the elevated CRP level (OR $=3.54,95 \%$ CI: 1.273-6.944, $P<0.05)$, the elevated IFN- $\gamma$ level $(\mathrm{OR}=1.50,95 \%$ CI: 1.712 8.629, $P<0.05)$, the elevated IL-6 level (OR $=4.55,95 \%$ CI: $3.175-19.210, P<0.01$ ), and the elevated Lp-PLA2 level (OR $=3.54,95 \%$ CI: $2.022-10.320, P<0.01)$ were independent risk factors for $\mathrm{KD}$ with CAL.
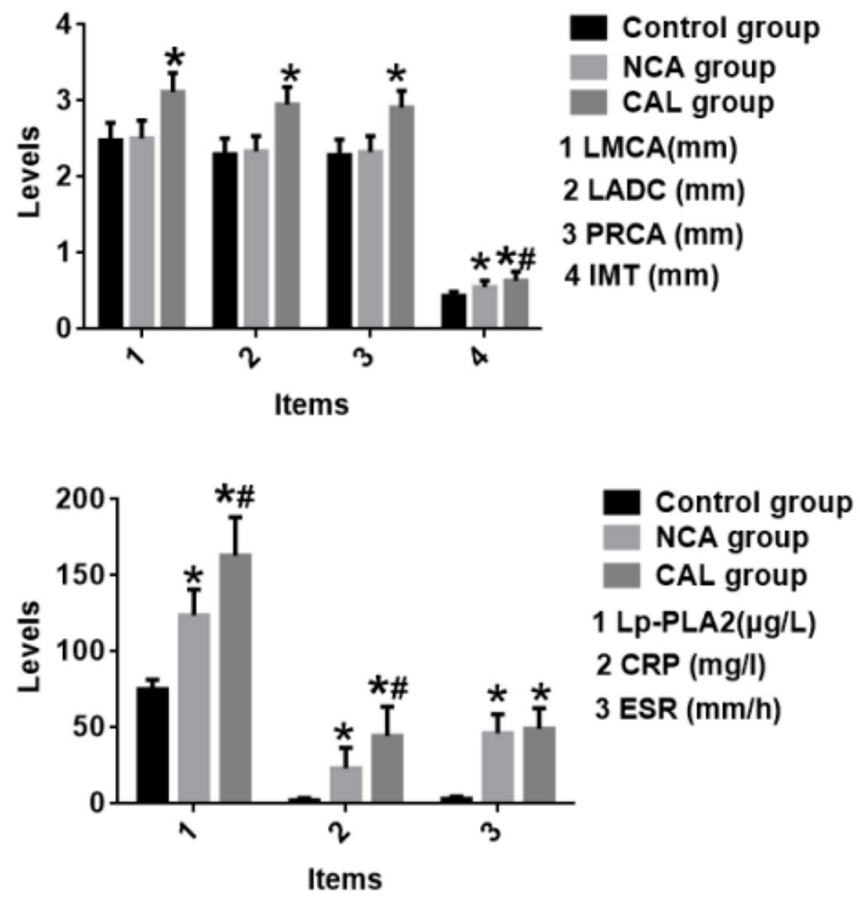

Correlation analysis: Correlation analysis showed that Lp-PLA2 level was positively correlated with the levels of IFN- $\gamma$, IL-6, and CRP in the CAL group and NCA group (respectively, all $P<0.01$ ). In addition, a similar correlation also was described between Lp-PLA2 level and the diameters of LMCA, LADC and PRCA in the CAL group (respectively, all $P<0.01$ ). (Figure 4)

ROC curve analysis: The AUC of serum Lp-PLA2 in the diagnosis of $\mathrm{KD}$ was [0.891 (95\% CI: $0.798-0.983, P<0.01)$ ], and the sensitivity and specificity were $94.59 \%$ and $91.89 \%$, respectively. The AUC of Lp-PLA2 in the diagnosis of KD with CAL was [0.889 (95\% CI: 0.801-0.977, $P<0.01)$ ], and the

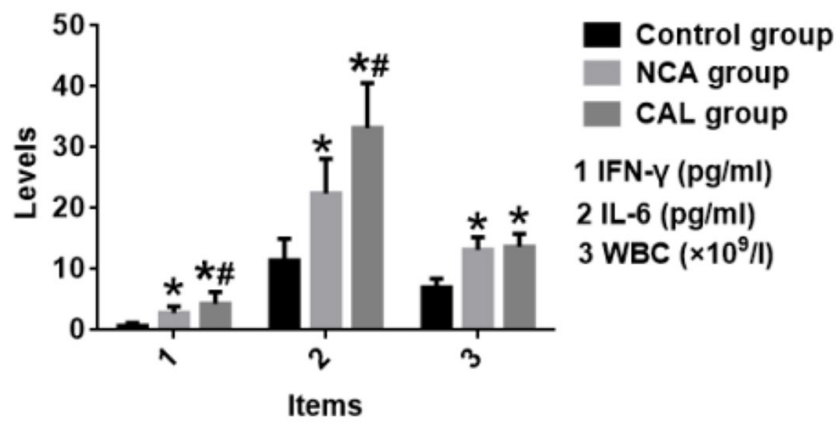

Figure 3. Laboratory analysis for KD. ${ }^{*} P$ value $<0.05$, compared with the control group (SNK-q test); $\# P$ value $<0.05$, compared with NCA group (SNK-q test). Lp-PLA2, Lipoprotein associated phospholipase A2; CAL, coronary artery lesions; NCA, normal coronary artery; IFN- $\gamma$, Interferon- $\gamma$; IL-6, Interleukin-6; ESR, erythrocyte sedimentation rate; CRP, C-reactive protein; LMCA, left main coronary artery; LADC, left anterior descending coronary artery; PRCA, right proximal coronary artery; IMT, carotid intima-media thickness

Table 4. Laboratory analysis for KD $\left({ }^{{ }} \pm s\right)$

\begin{tabular}{|c|c|c|c|c|}
\hline Lp-PLA2 ( $\mu \mathrm{g} / \mathrm{I})$ & $75.37 \pm 6.16$ & $123.67 \pm 17.29$ * & $163.02 \pm 25.33 * \#$ & $<0.01$ \\
\hline IL-6 (pg/ml) & $11.43 \pm 3.57$ & $22.45 \pm 5.71$ * & $33.19 \pm 7.38 * \#$ & $<0.01$ \\
\hline WBC $(\times 109 / 1)$ & $7.02 \pm 1.47$ & $13.18 \pm 2.07$ * & $13.73 \pm 2.09$ * & $<0.01$ \\
\hline $\operatorname{ESR}(\mathrm{mm} / \mathrm{h})$ & $3.63 \pm 1.49$ & $46.37 \pm 12.34$ * & $49.20 \pm 13.57$ * & $<0.01$ \\
\hline
\end{tabular}

$* P$ value $<0.05$, Compared with the control group; $\# P$ value $<0.05$, Compared with NCA group. CAL, coronary artery lesions; NCA, normal coronary artery; Lp-PLA2, Lipoprotein associated phospholipase A2; IFN- $\gamma$, Interferon- $\gamma$; IL-6, Interleukin-6; ESR, erythrocyte sedimentation rate; CRP, C-reactive protein 
sensitivity and specificity were $87.18 \%$ and $92.31 \%$, respectively. (Figure 5) It is suggested that serum Lp-PLA2 level is of clinical significance in the diagnosis of $\mathrm{KD}$ or $\mathrm{KD}$ with CAL.

\section{DISCUSSION}

Kawasaki disease is an acute inflammatory small and medium artery vasculitis that is common in children under 5 years old [Nakamura 2010]. The pathogenesis of KD still is unclear. Previous studies have shown that lipid metabolism disorder may be related to the occurrence and development of $\mathrm{KD}$ and can cause cholesterol deposition in vascular wall and promote the formation of atherosclerotic plaque. The change of lipid metabolism plays a leading role in the development of atherosclerosis. Adipose tissue can secrete a variety of bioactive inflammatory cytokines, which can promote the inflammatory process of blood vessels in children with KD [Biscetti 2019;
Poznyak 2020]. Related reports showed that KD patients had abnormal risk factors of atherosclerosis, and increased IMT thickness was observed in $\mathrm{KD}$ patients with $\mathrm{CAL}$, which is considered to be the initial pathological change secondary to atherosclerosis. Scholars suspect that the observed increase in IMT may be caused by diffuse vasculitis, involving coronary and noncoronary arteries in the acute phase [Noto 2009; Liu 2007]. In Meena's study [Meena 2014], IMT was measured at least one year after $\mathrm{KD}$ onset, and the results showed that IMT in the case group was higher than that in the normal control group. It is suggested that the vascular wall damage is persistent in $\mathrm{KD}$ children, and there is still persistent vascular inflammation in the recovery period of $\mathrm{KD}$. In this study, IMT in the control group, NCA group, and CAL group showed a gradually increasing trend. It is suggested that the IMT of KD children is different from that of normal children of the same age group, especially in the convalescent period, and the IMT is related to the severity of $\mathrm{KD}$.
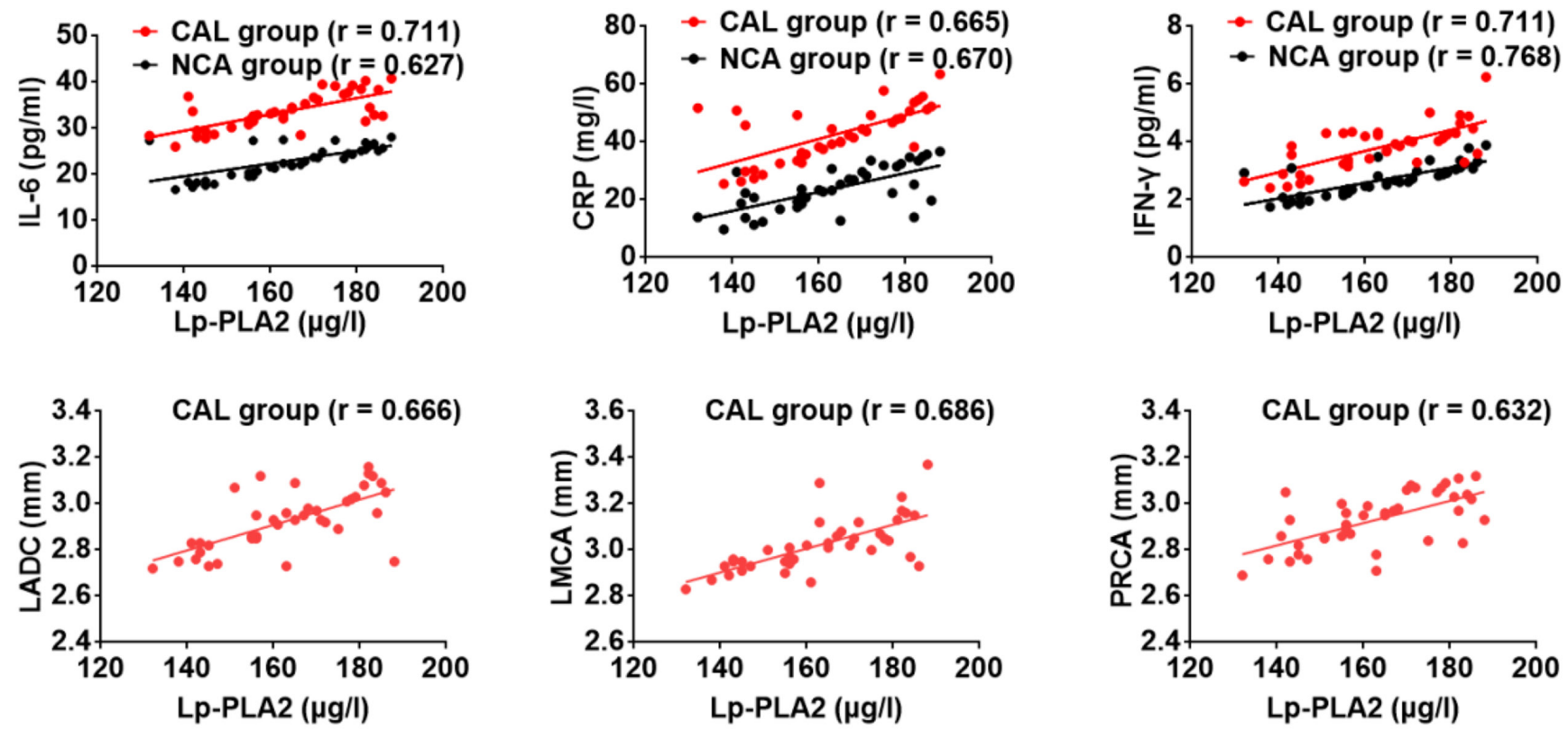

Figure 4. Correlation analysis of Lp-PLA2 (all $P<0.01$ ). Lp-PLA2, Lipoprotein associated phospholipase A2; CAL, coronary artery lesions; NCA, normal coronary artery; IFN- $\gamma$, Interferon- $\gamma$; IL-6, Interleukin-6; ESR, erythrocyte sedimentation rate; CRP, C-reactive protein; LMCA, left main coronary artery; LADC, left anterior descending coronary artery; PRCA, right proximal coronary artery; IMT, carotid intima-media thickness
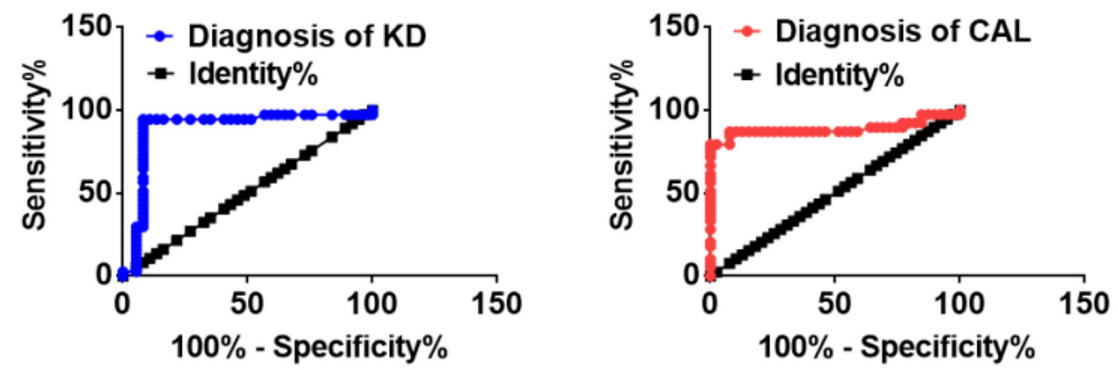

Figure 5. ROC curve of serum Lp-PLA2 in the diagnosis of KD or KD with CAL. KD, Kawasaki disease; CAL, coronary artery lesions 
Lp-PLA2 is a newly discovered atherosclerosis related vascular endothelial injury factor. It has been reported that macrophages and neutrophils induce atherosclerotic plaque to secrete excessive Lp-PLA2 into the blood circulation [Macphee 2006]. Lp-PLA2 can specifically hydrolyze oxidized phospholipids in subintimal low density lipoprotein to produce pro-inflammatory substances, oxidized free fatty acids and lysophosphatidylcholine (LysoPC). LysoPC seems to represent most of the inflammatory effects derived from Lp-PLA2 protein. It can target endothelial cells, smooth muscle cells, monocytes/macrophages, $\mathrm{T}$ cells and neutrophils, and then affect cell viability, inflammatory cell release and functional response of endothelial cells and smooth muscle cells, and induce oxidative stress and immune response, thus promoting the occurrence of inflammatory reaction [Zalewski 2005]. Due to proinflammatory effect and high distribution in the blood for Lp-PLA2, it has been observed in epidemiological studies that the elevated Lp-PLA2 level in plasma is associated with many vascular diseases [Munzel 2009; Siddiqui 2018; Acosta 2017; Lehtinen 2017]. Compared with other inflammatory markers, Lp-PLA2 is a cardiovascular specific inflammatory marker and can be used as an independent predictor of cardiovascular events [Chen 2017]. This study found that the serum Lp-PLA2 level in KD children was significantly higher than in the control group, and the increase was more significant in the CAL group. In addition, in this study, Lp-PLA2 level was positively correlated with IMT. It is suggested that Lp-PLA2 may be involved in the vascular lesions of $\mathrm{KD}$ children, which may be related to the pathological mechanism of atherosclerosis. The increase of Lp-PLA2 concentration may increase the risk of CAL in KD children.

Most researchers believe that immune system disorder and $T$ cell dysfunction are the main pathophysiological characteristics of KD patients [Matsubara 2005]. Studies have found that inflammatory cytokines, such as IFN- $\gamma$ and IL- 6 are involved in the pathogenesis of $\mathrm{KD}$ and related to the occurrence of coronary heart disease for KD [Wang 2020; Kobayashi 2004]. In the acute phase of KD, IL-6 signaling pathways are activated by monocytes in patients' blood [Leung 1986]. In the $\mathrm{KD}$ mouse model induced by cell wall extract of Lactobacillus casei, the increase of IL-6 has been confirmed to interrelate with the pathogenesis of CAL [Yandie 2019]. Another study has found that IFN- $\gamma$ plays a synergistic role in the occurrence of coronary heart disease. The increase of IFN- $\gamma$ level and its gene mutation may concern the occurrence of coronary heart disease [Wang 2019]. Another recent study showed that the IFN- $\gamma$ level of KD patients before intravenous immunoglobulin treatment is significantly increased, and the IFN- $\gamma$ level of patients with CAL is higher than that of patients without CAL. Further analysis showed that IFN- $\gamma$ was related to the occurrence of coronary artery aneurysms in $\mathrm{KD}$ patients [Huang 2016]. A related report [Kobayashi 2006] considered that the incidence of coronary artery aneurysm in $\mathrm{KD}$ patients with strong positive CRP was significantly increased. In this study, the results of logistic regression analysis showed that the elevated levels of CRP, IFN- $\gamma$, IL-6, and Lp-PLA2 were independent risk factors for KD with CAL. Our further analysis showed that the levels of IFN- $\gamma$, CRP, and IL- 6 were significantly increased in KD children, and CAL children showed a significant increase trend, suggesting that IFN- $\gamma$, CRP and IL-6 were involved in the pathological progress of KD. Furthermore, our results showed Lp-PLA2 level was positively correlated with IFN- $\gamma$, CRP, and IL-6 levels in $\mathrm{KD}$ children, respectively. These were further confirmed that Lp-PLA2 induced vascular lesions in KD children via inflammatory response, and the increased level of Lp-PLA2 could promote the occurrence of CAL in children with KD.

Acute KD selectively involves coronary artery, although it is only one of several moderate artery diseases affecting children under 5 years old [Zhang 2020]. Acute KD vasculitis affects not only coronary arteries, but also other arteries of similar size. Many previous pathological studies have shown that acute $\mathrm{KD}$ is a kind of acute systemic vasculitis with multiple organs [Amano 1979; Naoe 1991; Takahashi 2007]. The typical CAL caused by KD is located in the proximal segment of the epicardial coronary artery. However, based on the available evidence, the details of its distribution are not always clear [Tsuda 2017]. At present, Lp-PLA2 has been used in the diagnosis and treatment of cardiovascular diseases. A study has shown that Lp-PLA2 concentration is closely related to plaque vulnerability [Kolodgie 2006]. In a prospective cohort study of patients undergoing carotid endarterectomy, the expression of Lp-PLA2 in carotid plaques of patients with cardiac events was higher than that of patients without cardiac events [Herrmann 2009]. Lp-PLA2, as a biomarker of oxidation and inflammation, is associated with increased risk of coronary artery disease [Kobayashi 2006]. Children with KD often have atypical signs and symptoms [Xue 2020]. Color Doppler echocardiography is an imaging technique used to identify coronary artery abnormalities and evaluate myocardial function. Coronary artery diameter is helpful in the diagnosis of $\mathrm{KD}$ and CAL risk stratification [McCrindle 2018]. In this study, the results of ultrasound examination in KD children showed that the diameters of LMCA, LADC, and PRCA in the CAL group were significantly higher than those in the NCA group and control group. A similar trend was observed in KD children with CAL at different ages. Our correlation analysis showed that the serum Lp-PLA2 level of KD children with CAL was positively correlated with the diameters of LMCA, LADC and PRCA, respectively.

Furthermore, the ROC curve analysis results showed that Lp-PLA2 had good sensitivity and specificity for the diagnosis of $\mathrm{KD}$ and $\mathrm{KD}$ with CAL, suggesting that the detection of Lp-PLA2 level could evaluate the severity of KD patients and predict the occurrence and progress of CAL.

\section{CONCLUSION}

Our results suggest that increased Lp-PLA2 level in KD children may be associated with the occurrence of $\mathrm{KD}$. Therefore, longitudinal Lp-PLA2 measurement can provide a valuable tool to identify early CAL in children with KD.

Limitations of the study: There are some limitations in the current study. First, this article did not analyze the blood 
lipid level of children with $\mathrm{KD}$, due to the disorder of lipid metabolism that may be related to the occurrence and development of KD [Biscetti 2019]. Second, the study of single index may have limitations in evaluating KD and CAL.

\section{ACKNOWLEDGEMENTS}

This study was approved by the Medical Ethics Committee of the First Affiliated Hospital of Xi'an Jiaotong University and the First People's Hospital of Xian Yang City. Informed consent was obtained by the guardians of all participants. The study was conducted in accordance with the Declaration of Helsinki. We thank Yanxun Kang from the First People's Hospital of Xian Yang City for his critical suggestions and comments on this manuscript.

\section{REFERENCES}

Acosta S, Taimour S, Gottsater A, et al. 2017. Lp-PLA2 activity and mass for prediction of incident abdominal aortic aneurysms: A prospective longitudinal cohort study. Atherosclerosis. 262: 14-18.

Amano S, Hazama F, Hamashima Y. 1979. Pathology of Kawa-saki disease: I. Pathology and morphogenesis of the vascular changes. Jpn Circ. J 43: 633-643.

Biscetti F, Nardella E, Bonadia N, et al. 2019. Association between plasma omentin-1 levels in type 2 diabetic patients and peripheral artery disease. Cardiovasc Diabetol. 1:74.

Chen AD, Wang CL, Qin Y, et al. 2017. The effect of Danshen extract on lipoprotein-associated phospholipase A2 levels in patients with stable angina pectoris: study protocol for a randomized controlled trial - the DOLPHIN study. Trials. 18: 606

Choi WG, Prasad M, Lennon R, et al. 2018. Chronic darapladib use does not affect coronary plaque composition assessed using multimodality intravascular imaging modalities: A randomized-controlled study. Coron Artery Dis. 2:104-113.

Hedrich CM, Schnabel A, Hospach T. 2018. Kawasaki Disease. Front Pediatr. 6: 198.

Herrmann J, Mannheim D, Wohlert C, et al. 2009. Expression of lipoprotein-associated phospholipase A2 in carotid artery plaques predicts long-term cardiac outcome. Eur Heart J. 23:2930-8.

Huang YH, Hsu YW, Lu HF, et al. 2016. Interferon-gamma Genetic Polymorphism and Expression in Kawasaki Disease. Medicine (Baltimore). $17: \mathrm{e} 3501$.

Kobayashi N, Mori M, Kobayashi Y, et al. 2004. Intravenous gammaglobulin therapy improves hypercytokinemia in the acute phase of Kawasaki disease. Mod Rheumatol. 14:447-452.

Kobayashi T, Inoue Y, Takeuchi K, et al. 2006. Prediction of intravenous immunoglobulin unresponsiveness in patients with Kawasaki disease. Circulation. 22:2606-2612.

Kolodgie FD, Burke AP, Skorija KS, et al. 2006. Lipoprotein-associated phospholipase A2 protein expression in the natural progression of human coronary atherosclerosis. Arterioscler Thromb Vasc Biol. 11:2523-9.

Lehtinen L, Vainio P, Wikman H, et al. 2017. PLA2G7 associates with hormone receptor negativity in clinical breast cancer samples and regulates epithelial-mesenchymal transition in cultured breast cancer cells. J Pathol Clin Res. 2:123-138.

Leung DY, Geha RS, Newburger JW. et al. 1986. Two monokines, interleukin 1 and tumor necrosis factor, render cultured vascular endothelial cells susceptible to lysis by antibodies circulating during Kawasaki syndrome. J. Exp. Med. 164:1958-1972.

Liu XQ, Huang GY, Liang XC, et al. 2007. The study of vascular lesion using ultrasound in children with a history of Kawasaki disease. Zhonghua Yi Xue Za Zhi. 30: 2117-20.

Macphee CH, Nelson J, Zalewski A. 2006. Role of lipoprotein-associated phospholipase $\mathrm{A} 2$ in atherosclerosis and its potential as a therapeutic target. Curr Opin Pharmacol. 2: 154-161.

Matsubara T, Ichiyama T, Furukawa S. 2005. Immunological profile of peripheral blood lymphocytes and monocytes/macrophages in Kawasaki disease. Clin. Exp. Immunol. 141: 381-387.

McCrindle BW, Cifra B. 2018. The role of echocardiography in Kawasaki disease. Int J Rheum Dis. 21:50-55.

McCrindle BW, Rowley AH, Newburger JW, et al. 2017. Diagnosis, treatment, and long-term management of Kawasaki disease: a scientific statement for health professionals from the American Heart Association. Circulation. 17: e927- e999.

Meena RS, Rohit M, Gupta A, et al. 2014. Carotid intima-media thickness in children with Kawasaki disease. Rheumatol Int, 8: 1117-1121.

Menikou S, Langford PR, Levin M. 2019. Kawasaki disease: the role of immune complexes revisited. Front Immunol. 10: 1156.

Munzel T, Gori T. 2009. Lipoprotein-associated phospholipase A(2), a marker of vascular inflammation and systemic vulnerability. Eur Heart J. 23: 2829-2831.

Nakamura Y, Yashiro M, Uehara R, et al. 2010. Epidemiologic features of Kawasaki disease in Japan: results of the 2007-2008 nationwide survey. J. Epidemiol. 20: 302-307.

Naoe S, Takahashi K, Masuda H, et al. 1991. Kawasaki disease. With particular emphasis on arterial lesions. Acta Pathol Jpn. 41:785-797.

Noto N, Okada T, Karasawa K, et al. 2009. Age-related acceleration of endothelial dysfunction and subclinical atherosclerosis in subjects with coronary artery lesions after Kawasaki disease. Pediatr Cardiol. 30: 262-268.

Poznyak AV, Zhang DW, Orekhova V, et al. 2020. A brief overview of currently used atherosclerosis treatment approaches targeting lipid metabolism alterations. Am J Cardiovasc Dis. 2:62-71.

Siddiqui MK, Kennedy G, Carr F, et al. 2018. Lp-PLA2 activity is associated with increased risk of diabetic retinopathy: a longitudinal disease progression study. Diabetologia. 6:1344-1353.

Takahashi K, Oharaseki T, Yokouchi Y, et al. 2007. Kawasaki disease arteritis and polyarteritis nodosa. Pathol Case Rev. 12:193-199.

Tsuda E, Tsujii N, Kimura K, et al. 2017. Distribution of Kawasaki Disease coronary artery aneurysms and the relationship to coronary artery diameter. Pediatr Cardiol. 38:932-940.

Wang HL, Zhang N, Li GR, et al. 2019. Proinflammatory cytokine IFN- $\gamma$, lncRNA BANCR and the occurrence of coronary artery disease. Life Sciences. 231:116510.

Wang Y, Qian SY, Yuan Y, et al. 2020. Do cytokines correlate with refractory Kawasaki disease in children? Clinica Chimica Acta. 506:222-227.

Xue M, WangJ. 2020. Utility of color Doppler echocardiography combined 
with clinical markers in diagnosis and prediction of prognosis of coronary artery lesions in Kawasaki disease. Exp Ther Med. 19:2597-2603.

Yandie Li, Qi Zheng, Lixia Zou, et al. 2019. Kawasaki disease shock syndrome: clinical characteristics and possible use of IL-6, IL-10 and IFN- $\gamma$ as biomarkers for early recognition. Pediatr Rheumatol Online J. 1:1.
Zalewski A, Macphee C. 2005. Role of lipoprotein-associated phospholipase A2 in atherosclerosis: biology, epidemiology, and possible therapeutic target. Arterioscler Thromb Vasc Biol. 5: 923-931.

Zhang DF, Liu LJ, Huang XP, et al. 2020. Insights Into Coronary Artery Lesions in Kawasaki Disease. Front Pediatr. 8:493. 